\title{
Dietary sugar restriction reduces hepatic de novo lipogenesis in adolescent boys with fatty liver disease
}

\author{
Catherine C. Cohen, ${ }^{1,2}$ Kelvin W. Li, ${ }^{3}$ Adina L. Alazraki, ${ }^{4,5}$ Carine Beysen, ${ }^{6}$ Carissa A. Carrier, ${ }^{7}$ Rebecca L. Cleeton, ${ }^{1}$ \\ Mohamad Dandan, ${ }^{3}$ Janet Figueroa, ${ }^{1}$ Jack Knight-Scott, ${ }^{5}$ Cynthia J. Knott, ${ }^{8}$ Kimberly P. Newton, ${ }^{7,9}$ Edna M. Nyangau, ${ }^{3}$ \\ Claude B. Sirlin, ${ }^{10}$ Patricia A. Ugalde-Nicalo, ${ }^{7,9}$ Jean A. Welsh, ${ }^{1,11}$ Marc K. Hellerstein, ${ }^{3}$ Jeffrey B. Schwimmer, ${ }^{7,9}$ and Miriam B. Vos ${ }^{1,11}$ \\ 1Department of Pediatrics, School of Medicine, Emory University, Atlanta, Ceorgia, USA. ²Department of Pediatrics, School of Medicine, University of Colorado Denver, Aurora, Colorado, USA. ${ }^{3}$ Department \\ of Nutritional Sciences and Toxicology, University of California, Berkeley, Berkeley, California, USA. ${ }^{4}$ Department of Radiology, School of Medicine, Emory University, Atlanta, Ceorgia, USA. ${ }^{5}$ Department of \\ Radiology, Children's Healthcare of Atlanta, Atlanta, Ceorgia, USA. ${ }^{6}$ Fluxbio, San Mateo, California, USA. 'Department of Pediatrics, School of Medicine, UCSD, La Jolla, California, USA. ${ }^{8}$ Altman Clinical and \\ Translational Research Institute, School of Medicine, UCSD, La Jolla, California, USA. ํDepartment of Gastroenterology, Rady Children's Hospital San Diego, San Diego, California, USA. ${ }^{\circ}$ Liver Imaging Group, \\ Department of Radiology, UCSD, La Jolla, California, USA. "Department of Gastroenterology, Hepatology, and Nutrition, Children's Healthcare of Atlanta, Atlanta, Georgia, USA
}

BACKGROUND. Hepatic de novo lipogenesis (DNL) is elevated in nonalcoholic fatty liver disease (NAFLD). Improvements in hepatic fat by dietary sugar reduction may be mediated by reduced DNL, but data are limited, especially in children. We examined the effects of 8 weeks of dietary sugar restriction on hepatic DNL in adolescents with NAFLD and correlations between DNL and other metabolic outcomes.

METHODS. Adolescent boys with NAFLD $(n=29)$ participated in an 8-week, randomized controlled trial comparing a diet low in free sugars versus their usual diet. Hepatic DNL was measured as percentage contribution to plasma triglyceride palmitate using a 7-day metabolic labeling protocol with heavy water. Hepatic fat was measured by magnetic resonance imaging-proton density fat fraction.

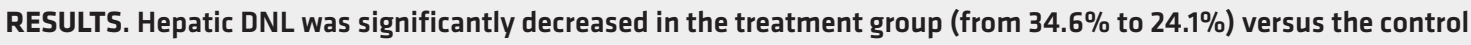
group (33.9\% to $34.6 \%$ ) (adjusted week 8 mean difference: $-10.6 \%$ [95\% Cl: $-19.1 \%,-2.0 \%]$ ), which was paralleled by greater decreases in hepatic fat ( $25.5 \%$ to $17.9 \%$ vs. $19.5 \%$ to $18.8 \%$ ) and fasting insulin ( 44.3 to 34.7 vs. 35.5 to $37.0 \mu \mathrm{IU} /$ $\mathrm{mL}$ ). Percentage change in DNL during the intervention correlated significantly with changes in free-sugar intake ( $r=$ $0.48, P=0.011)$, insulin $(r=0.40, P=0.047)$, and alanine aminotransferase (ALT) $(r=0.39, P=0.049)$, but not hepatic fat $(r=0.13, P=0.532)$.

CONCLUSION. Our results suggest that dietary sugar restriction reduces hepatic DNL and fasting insulin, in addition to reductions in hepatic fat and ALT, among adolescents with NAFLD. These results are consistent with the hypothesis that hepatic DNL is a critical metabolic abnormality linking dietary sugar and NAFLD.

TRIAL REGISTRY. ClinicalTrials.gov NCT02513121.

FUNDING. The Nutrition Science Initiative (made possible by gifts from the Laura and John Arnold Foundation, Ambrose Monell Foundation, and individual donors), the UCSD Altman Clinical and Translational Research Institute, the NIH, Children's Healthcare of Atlanta and Emory University's Children's Clinical and Translational Discovery Core, Children's Healthcare of Atlanta and Emory University Pediatric Biostatistical Core, the Georgia Clinical and Translational Science Alliance, and the NIH National Institute of Diabetes, Digestive, and Kidney Disease. 


\section{Related Commentary: https://doi.org/10.1172/JCI154645}

ROLE OF FUNDING SOURCE. The funders assisted with protocol development by convening a group of subject matter experts to discuss study design. The final design was developed by the authors and approved by the funder. The funder otherwise had no role in data collection and analysis, decision to publish, or preparation of the manuscript. Conflict of interest: JBS has received research support from Galmed, Intercept, Cenfit, and Seraphina. CBS has served as a consultant representative of the University of California Regents for GE Healthcare, Bayer, Boehringer Ingelheim, AMRA, Fulcrum Therapeutics, Medscape, and Resoundant; has served on scientific advisory boards for AMRA, Guerbet, and VitualScopics; is receiving research grants from ACR Innovation, Bayer, Gilead, GE Healthcare, General Electric, Philips, and Siemens; has served as a consultant for AMRA, Boehringer Ingelheim, Epigenomics, and Guerbet; has served on the speaker's bureau for Resoundant and Ceneral Electric; has laboratory service agreements with Enanta, Genzyme, Gilead, Icon Medical Imaging, Intercept, Janssen, NuSirt, Shire, Synageva, Takeda, and VitualScopics; has received royalties from Wolters Kluwer for educational materials; serves on the advisory board for Quantix Bio; and owns stock options in Livivos. MBV has received grant support from Resonance Health, Immuron, Gemphire, Shire, and Target Pharmasolutions; and personal fees from AMRA, Immuron, Intercept, Target Pharmasolutions, Shire, Bristol-Myers Squibb, Boehringer Ingelheim, and Axcella Health.

Copyright: @ 2021, American Society for Clinical Investigation.

Submitted: May 4, 2021; Accepted: October 27, 2021; Published: December 15, 2021. Reference information: J Clin Invest. 2021;131(24):e150996. https://doi.org/10.1172/JCl150996.

\section{Introduction}

The prevalence of pediatric nonalcoholic fatty liver disease (NAFLD), defined by hepatic fat accumulation in the absence of excess alcohol intake, has increased considerably over recent decades $(1,2)$. This trend has occurred in association with the rise of obesity in childhood and adolescence and is projected to have major public health consequences because NAFLD drives earlyonset cardiometabolic abnormalities, including insulin resistance (3-5), dyslipidemia (6), and atherosclerosis markers (7). Children with NAFLD, especially those with the more severe form of the disease, nonalcoholic steatohepatitis (NASH), are at higher risk for developing prediabetes and type 2 diabetes $(8,9)$, and for progressing to end-stage liver disease (10).

Hepatic fat accumulates when rates of fatty acid uptake and production exceed their oxidation and export by the liver. Increased hepatic de novo lipogenesis (DNL), the process by which excess non-lipid energy substrates are converted to fatty acids, may be a critical metabolic abnormality within this system, given the markedly elevated rates of DNL observed in adults with NAFLD. Lambert et al. reported a $26 \%$ contribution from DNL to triglyceride (TG) palmitate in adults with NAFLD, which was a 3-fold elevation compared with adults without NAFLD (11). More recently, Smith et al. reported even higher values of hepatic DNL in adults with obesity and NAFLD (40\% contribution from DNL to palmitate) using a longer-term protocol that allowed more complete labeling to be attained in slowly turning over liver TG stores (12). In these studies, hepatic DNL was correlated with hepatic fat $(11,12)$ as well as 24 -hour plasma glucose and insulin and systemic and hepatic insulin resistance (measured by 2-stage glucose clamp technique) (12), and fell with weight loss (12). These findings suggest a central role of DNL in hepatic fat accumulation and of hyperinsulinemia in stimulating hepatic DNL. Excess sugar intake also appears to be a strong risk factor for NAFLD (13-15), which may be mediated by hepatic DNL. Experimental studies in adults have shown that shifting to high-sugar diets, especially diets containing fructose, increases both hepatic DNL (16-21) and hepatic fat (21), even in the absence of weight gain (21). While data are more limited in children, a 9-day, noncontrolled study of 41 children with obesity (9-18 years old) showed that isocaloric dietary fructose restriction results in significantly reduced DNL and hepatic fat (22). However, they used a brief, 8-hour labeling protocol to measure DNL. Due to these limitations, additional data are needed to confirm that sugar reduction improves NAFLD by reducing DNL in children.

The objective of this study was to test the effect of longer-term dietary sugar restriction on hepatic DNL in adolescent boys with NAFLD (11-16 years old) who participated in an 8-week randomized, controlled treatment study testing the effects of a lowfree-sugar diet compared with their usual diet (23). A total of 40 participants completed the treatment study, among whom 29 participants completed a stable isotope tracer protocol at baseline and/or at study completion to assess hepatic DNL (Figure 1). The primary aim was to examine whether the 8-week diet treatment reduces hepatic DNL, measured with an optimal labeling paradigm, and the secondary aim was to examine associations between change in DNL and change in hepatic fat, as measured by magnetic resonance imaging-proton density fat fraction (MRIPDFF), and other secondary outcomes, including fasting insulin and glucose concentrations, blood lipids, and liver enzymes.

\section{Results}

Study population. A flow diagram for the participants included in the present study is shown in Figure 1, and an overview of the protocol and procedures involved is shown in Figure 2. A total of 29 participants completed the hepatic DNL assessment at baseline and the characteristics of this sample are summarized in Table 1. Mean age was similar in the control group $(13.3 \pm 1.9$ years) versus the treatment group $(12.6 \pm 1.9$ years; $P=0.34)$. None of the sociodemographic, laboratory, or anthropometric variables differed between groups at baseline, except BMI $z$ score, which was lower in the control versus treatment group $(2.09 \pm 0.48$ vs. $2.46 \pm 0.23$, respectively; $P=0.012)$. From baseline to week 8 , the change in free-sugar intake in this subsample was similar to the change in free-sugar intake observed in the full sample, as reported by Schwimmer et al. (23). Based on mixed models adjusted for study site, the treatment group experienced a greater decreases in free-sugar intake (from 9.9\% to $1.1 \%$ ) compared with the control group (from 13.1\% to 11.8\%) (Supplemental Table 1; supplemental material available online with this article; https://doi.org/10.1172/JCI150996DS1). The treatment group also experienced a greater decrease in hepatic fat measured by MRI-PDFF (from $25.5 \%$ to $17.9 \%$ ) compared with the control group (from $19.5 \%$ to $18.8 \%$ ), fasting insulin (from 44.3 to $34.7 \mu \mathrm{IU} / \mathrm{mL}$ in the treatment group compared with 35.5 to 37.0 $\mu \mathrm{IU} / \mathrm{mL}$ in the control group), and other secondary outcomes, including fasting glucose, TGs, total and LDL cholesterol, and liver enzymes (alanine aminotransferase [ALT], aspartate aminotransferase [AST], and $\gamma$-glutamyl transferase [GGT]) (all $P<$ 0.05; Supplemental Table 2). The treatment group also experi- 


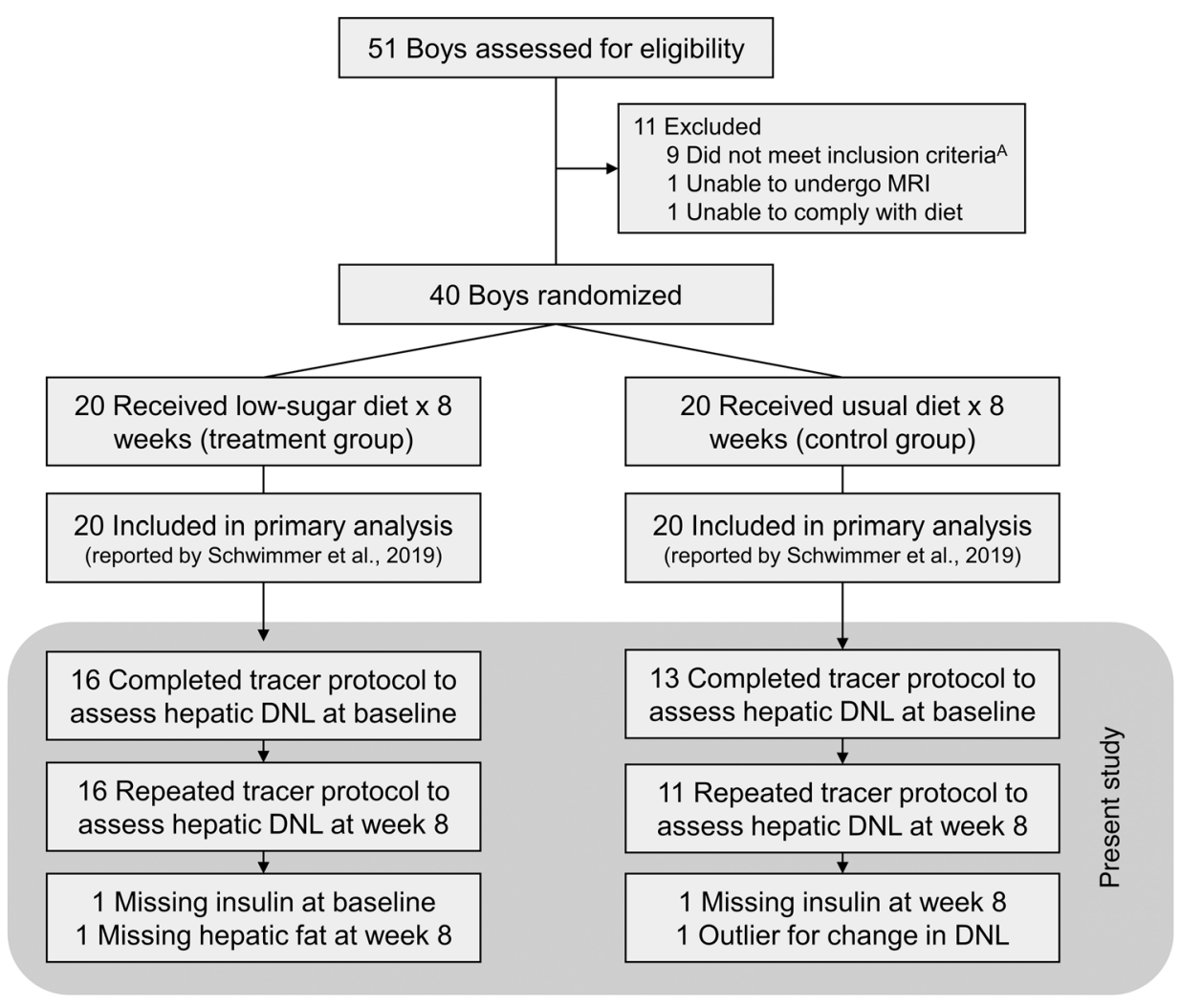

Figure 1. Flow diagram of participants included in the present analysis. This figure was adapted from the flow diagram for the original intervention trial by Schwimmer et al. (23) and expanded on. ${ }^{A}$ Based on alanine aminotransferase level, the magnetic resonance imaging-proton density fat fraction percentage, or diagnosis of diabetes at baseline. Abbreviations: MRI, magnetic resonance imaging. DNL, de novo lipogenesis.

Associations between changes in hepatic DNL and changes in secondary variables. Supplemental Table 3 summarizes the mean percentage change from baseline to week 8 for hepatic DNL, hepatic fat (MRI-PDFF), and the secondary metabolic outcomes in the full sample and by treatment group. For these analyses, we could only include participants with complete data at both time points for each variable in order to calculate percentage change. Therefore, we excluded 2 participants in the control group who were missing DNL data at week 8 . In relevant analyses, we also excluded 1 participant in the treatment group who was missing MRI-PDFF data at week 8 and 1 participant in the treatment group who was missing insulin data at baseline. In addition, we excluded 1 outlier participant in the control group who experienced a $480 \%$ increase in DNL from baseline to week 8. Most treatment group participants experienced decreases in key variables, including free-sugar intake, hepatic fat (MRIPDFF), fasting insulin, and ALT after 8 weeks, paralleling the decreases in hepatic DNL, whereas in the control

enced weight loss compared with the control group, although the magnitude of the weight loss in the treatment group was relatively small (91.1 to $89.7 \mathrm{~kg}$, Supplemental Table 2).

Effect of the 8-week dietary sugar restriction on hepatic DNL. At baseline, the mean \pm SD for hepatic DNL (measured as the percentage contribution from the DNL pathway to palmitate) was similar in the control group $(33.3 \% \pm 13.0 \%)$ versus the treatment group $(34.6 \% \pm 9.4 \% ; P=0.76)$. All but 2 participants in the control group completed the repeat labeling protocol at study completion. Based on mixed models adjusted for study site, the mean decrease in DNL from baseline to week 8 was greater for the treatment group (from $34.6 \%$ to $24.1 \%$ ) compared with the control group (from $33.9 \%$ to $34.6 \%$ ). The adjusted week 8 mean difference, based on mixed models adjusted for site and baseline, was $-10.6 \%$ (95\% CI: $-19.1 \%$, $-2.0 \%$; Table 2). Individual changes in hepatic DNL from baseline to week 8 are shown in Figure 3. We also examined whether results differed if we adjusted for percentage weight change during the intervention as an additional covariate in mixed models and found that the estimated mean changes in DNL in the treatment and control group were similar compared to the estimates in each group without adjusting for percentage weight change above (from $35.9 \%$ to $25.4 \%$ in the treatment group and from $33.3 \%$ to $32.9 \%$ in the control group in mixed models adjusted for study site and percentage weight change). The adjusted week 8 mean difference in DNL between treatment groups was also similar, but attenuated slightly (-7.9\% [95\% CI: $-16.0 \%, 0.2 \%], P=0.055)$. group, the directionality of changes was inconsistent (Supplemental Figure 1). We next examined correlations between percentage changes in hepatic DNL and percentage changes in these secondary variables during the intervention. In the full sample, change in DNL was significantly correlated with changes in free-sugar intake $(r$ [95\% CI]: 0.48 [0.12, 0.73]), ALT (0.39 [0.01, 0.67]), insulin $(0.40$ [0.01, 0.69]), and weight $(0.45$ [0.08, 0.72]), but not with change in hepatic fat (MRI-PDFF) $(0.13$ [-0.28, 0.50]) (Table 3). Scatter plots showing correlations between DNL and select secondary variables are shown in Figure 4. In analyses stratified by treatment group, these associations were similar, if not greater in magnitude, in the treatment group specifically, but were less precise (wider CIs) and some crossed the null ( $r$ [95\% CI]: 0.42 $[-0.09,0.76]$ for free-sugar intake, $0.51[0.02,0.80]$ for ALT, 0.30 $[-0.25,0.71]$ for insulin, and $0.40[-0.12,0.75]$ for weight; Table 3 and Figure 4).

\section{Discussion}

This study was the first to our knowledge to examine in a pediatric sample (adolescent boys with NAFLD) the changes in hepatic DNL, measured by stable isotope tracers, after participating in a longer-term (8 week), low-free-sugar dietary treatment protocol. Specifically, hepatic DNL was measured using a 7-day labeling protocol, which more accurately assesses the contribution of DNL to the slowly turning over storage pool of TG in the liver $(11,12,24)$. Because the longitudinal design 


\begin{tabular}{|c|c|c|c|c|c|c|c|c|}
\hline & & \multicolumn{2}{|c|}{ Labeling period \#1 } & & & \multicolumn{2}{|c|}{ Labeling period \#2 } & \\
\hline Assessment: & Day -14 & Day -7 & Day -6 to -1 & $\begin{array}{c}\text { Day } 0 \\
\text { (Baseline) }\end{array}$ & Day 28 & Day 47 & Day 48 to 55 & $\begin{array}{c}\text { Day } 56 \\
\text { (Week 8) }\end{array}$ \\
\hline DNL tracers $\left({ }^{2} \mathrm{H}_{2} \mathrm{O}\right)$ & & Started & $\longrightarrow$ & Stopped & & Started & & Stopped \\
\hline Fasting blood draw & & & & $\mathrm{X}$ & $\mathrm{X}$ & & & $\mathrm{x}$ \\
\hline Dried blood spot & & & & $x$ & & $X$ & & $\mathrm{X}$ \\
\hline Imaging (MRI) & $X$ & & & & $\mathrm{X}$ & & & $x$ \\
\hline Diet intervention & & & & Started & & & & Stopped \\
\hline
\end{tabular}

Figure 2. Clinical trial design and procedures. Depicted is the timing of the DNL stable isotope tracer protocol ( $50 \mathrm{~mL}$ of ${ }^{2} \mathrm{H}_{2} \mathrm{O}$ two times a day for 7 days), fasting blood draws, dried blood spot collection, and magnetic resonance imaging (MRI) throughout the duration of the intervention. All dried blood spots were collected at the same time as the fasting blood draw, except on day 47 (start of the second labeling period) when only a dried blood spot was collected to correct for the residual isotopic enrichment from the baseline labeling period. The purpose of the dried blood spot was to limit the number of blood draws.

required that the labeling protocol be repeated relatively quickly, it was necessary to correct for residual labeled fats from the baseline assessment that had yet not washed out of subjects. We showed that, after this correction, 8 weeks of dietary sugar restriction in adolescent boys with NAFLD decreased the DNL measured over a 7-day period by nearly one-third (from $34.6 \%$ to $24.1 \%$ ) compared with their usual diet. This finding was similar, but slightly attenuated after adjusting for weight change during the intervention. We also found that the change in DNL was directly correlated with changes in free-sugar intake, fasting insulin, and ALT during the intervention, although we did not find a correlation with change in hepatic fat (MRI-PDFF). Overall, these findings are consistent with the hypothesis that dietary free-sugar restriction is a strategy for reducing hepatic DNL, which in turn is beneficial for other metabolic outcomes in pediatric NAFLD.

Hepatic DNL rates are much higher in individuals with NAFLD/NASH compared with lean subjects without fatty liver

\section{Table 1. Characteristics of the sample according to treatment group}

\begin{tabular}{|c|c|c|c|c|c|}
\hline \multirow[b]{2}{*}{ Variable } & \multicolumn{2}{|c|}{ Control group $(n=13)$} & \multicolumn{3}{|c|}{ Treatment group $(n=16)$} \\
\hline & Mean or No. & SD or $\%$ & Mean or No. & SD or $\%$ & $P$ value ${ }^{A}$ \\
\hline Age at screening (yrs) & 13.31 & 1.93 & 12.63 & 1.86 & 0.34 \\
\hline Income of household & & & & & 0.24 \\
\hline$\$ 15,000-\$ 29,999$ & 5 & $38.5 \%$ & 2 & $12.5 \%$ & \\
\hline$\$ 30,000-\$ 49,999$ & 3 & $23.1 \%$ & 6 & $37.5 \%$ & \\
\hline Race/ethnicity & & & & & 0.56 \\
\hline Hispanic & 13 & $100 \%$ & 14 & $87.5 \%$ & \\
\hline Non-Hispanic White & 0 & $0 \%$ & 2 & $12.5 \%$ & \\
\hline \multicolumn{6}{|l|}{ Laboratory values } \\
\hline Fasting glucose (mg/dL) & 85.54 & 11.34 & 91.19 & 10.41 & 0.17 \\
\hline Total cholesterol (mg/dL) & 163.08 & 30.21 & 163.56 & 43.42 & 0.97 \\
\hline LDL cholesterol (mg/dL) & 100.39 & 25.40 & 101.81 & 35.72 & 0.90 \\
\hline HDL cholesterol (mg/dL) & 39.46 & 6.73 & 40.19 & 7.70 & 0.79 \\
\hline Triglycerides $(\mathrm{mg} / \mathrm{dL})^{\mathrm{B}}$ & 177 & $92-181$ & 131 & $67-214.5$ & 0.66 \\
\hline \multicolumn{6}{|l|}{ Anthropometrics } \\
\hline BMI z score & 2.09 & 0.48 & 2.46 & 0.23 & 0.01 \\
\hline Waist circumference (cm) & 102.70 & 14.73 & 110.71 & 12.67 & 0.13 \\
\hline Hip circumference (cm) & 103.37 & 12.90 & 110.56 & 11.24 & 0.13 \\
\hline Hepatic steatosis (\%) ${ }^{\mathrm{B}}$ & 17 & $15.1-25$ & 25.3 & $17.2-29.6$ & 0.15 \\
\hline
\end{tabular}

${ }^{A} P$ values were calculated using 2-tailed $t$ test for continuous variables or $\chi^{2}$ test for categorical variables. ${ }^{B}$ Reporting medians and IQRs due to skewed distribution; $P$ values calculated using 2-tailed, nonparametric Mann-Whitney $U$ test. ALT, alanine aminotransferase; AST, aspartate aminotransferase; GGT, $\gamma$-glutamyl transferase. 
Table 2. Estimates for hepatic DNL at baseline and week 8 by treatment group

\begin{tabular}{|c|c|c|c|}
\hline & Control group $(n=13)^{A}$ & Treatment group $(n=16)$ & \\
\hline Time point & LS mean $(95 \% \mathrm{CI})^{\mathrm{B}}$ & LS mean $(95 \% \mathrm{Cl})^{\mathrm{B}}$ & Adj. week 8 mean difference ${ }^{c}$ \\
\hline Baseline & $33.9 \%(27.7 \%, 40.1 \%)$ & $34.6 \%(29.0 \%, 49.1 \%)$ & \\
\hline Week 8 & $34.6 \%(28.1 \%, 41.0 \%)$ & $24.1 \%(18.8 \%, 29.4 \%)$ & $-10.6 \%(-19.1 \%,-2.0 \%)$ \\
\hline
\end{tabular}

disease $(11,12,24,25)$. It is increasingly accepted based on this evidence, along with well-established regulatory pathways in the liver linking DNL to inhibition of fatty acid oxidation through the shared metabolite malonyl-CoA (26), that DNL is an important component in the pathogenesis of NAFLD. Consistent with this model, fasting DNL values at baseline were around 34\% in adolescent boys with biopsy-proven NAFLD studied here, which is similar to values reported in adults with obesity and NAFLD (38\%; ref. 12) or NASH (43\%; ref. 24), but higher than DNL values reported in lean adults (11\%; ref. 12) and values reported in adolescents who are overweight/obese, but without NAFLD (approximately $5 \%-10 \%$; ref. 27 ). Thus, our data support the notion that children with NAFLD who are consuming high-sugar diets have high rates of hepatic DNL, which are similar to values that have been reported in adults with NAFLD. Furthermore, as described in the Methods section below, we found residual label enrichment in plasma palmitate from the baseline labeling period when we began the second labeling period on day 47, which indicates that, in addition to having high fasting DNL, the boys with NAFLD in this study also have a slow turnover of hepatic lipids.

Our finding that hepatic DNL was reduced in the treatment group is consistent with findings from a study by Schwarz et al.,

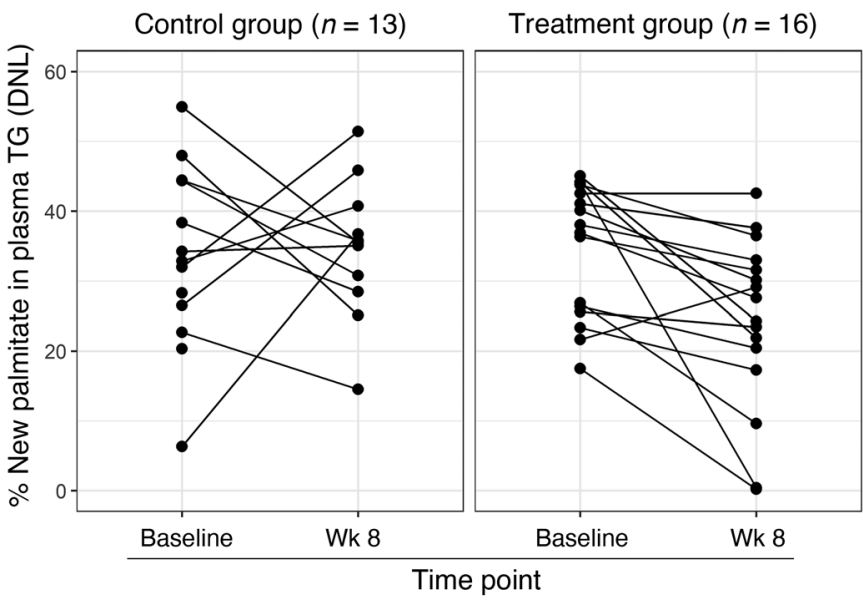

Figure 3. Individual changes in de novo lipogenesis (DNL) in adolescent boys with NAFLD from baseline to week 8 by treatment group. In the control group $(n=13)$, participants consumed their usual diet for 8 weeks. In the treatment group $(n=16)$, participants were provided a diet that was low in free sugars for 8 weeks. In the control group, 2 participants did not complete the hepatic DNL protocol at week 8 , but their baseline hepatic DNL is still included in this figure. which showed that, in 41 children with obesity and high habitual sugar consumption at baseline, 9 days of dietary fructose restriction significantly reduces fractional DNL, from a DNL area under the curve of $68.4 \%$ at baseline to $29.7 \%$ on day 10 (28). Further, the change in DNL was similar in the children who did not lose weight, which aligns with our pattern of findings. In that study, a brief 8-hour labeling protocol was used, compared with the longer labeling protocol in the present study. Also, in the above study, only fructose was restricted, while in the present study, all types of free sugar were restricted in the diet treatment group. Despite this difference, it is likely that the effect on DNL that we observed could be, at least in part, explained by the removal of dietary fructose both as an added sugar and in juice. This hypothesis is based on adult studies showing that fructose consumption is more strongly associated with DNL than other monosaccharides, such as glucose (18), and has a dose-dependent effect on DNL that is likely related to the metabolism of fructose in liver $(20,29)$. In particular, while glucose has a more systemic initial metabolic uptake $(30,31)$, fructose is primarily metabolized in the liver (32), where it contributes carbon substrates to hepatic pyruvate, acetyl-CoA, and DNL, and may indirectly stimulate DNL by transcriptional regulation via carbohydrate response element-binding protein and sterol regulatory element-binding protein $(33,34)$.

In addition to direct effects of dietary sugars on hepatic DNL, there may also be indirect effects related to changes in insulin sensitivity and exposure. While in our primary analysis (23) the low-free-sugar dietary intervention was not significantly associated with reductions in fasting insulin levels, this may be because insulin data were missing in nearly half of the samples at week 8 . In the present analysis, we measured insulin in stored samples from baseline and week 8 for participants who were previously missing insulin data at week 8 and, with these new data, showed that the diet treatment was associated with reduced fasting insulin levels (Supplemental Table 2). This finding of reduced fasting insulin concentrations in the absence of significant weight loss is an additional important result from this study, representing another metabolic benefit from reducing sugar intake in adolescents with NAFLD. Further, in this study, we showed that there were direct correlations between change in insulin during the intervention, as well as change in free-sugar intake, and change in hepatic DNL. This aligns with findings from a study by Smith et al., which showed that, in adults with and without NAFLD, hepatic DNL is strongly and directly correlated with 24-hour insulin and glucose concentrations and inversely correlated with systemic and hepatic insulin resistance (12). Collectively, these 
Table 3. Correlations between percentage changes in key secondary variables and percentage change in hepatic DNL in the full sample and stratified by treatment group

\begin{tabular}{|c|c|c|c|c|c|c|}
\hline \multirow[b]{2}{*}{$\%$ Change variable } & \multicolumn{2}{|c|}{ Full sample $(n=26)$} & \multicolumn{2}{|c|}{ Control group $(n=10)^{\mathrm{A}}$} & \multicolumn{2}{|c|}{ Treatment group $(n=16)$} \\
\hline & $r(95 \% \mathrm{Cl})^{\mathrm{B}}$ & $P$ & $r(95 \% \mathrm{Cl})^{\mathrm{B}}$ & $P$ & $r(95 \% \mathrm{Cl})^{\mathrm{B}}$ & $P$ \\
\hline Free sugar (\% TEl/d) & $0.48(0.12,0.73)$ & 0.011 & $0.47(-0.23,0.85)$ & 0.178 & $0.42(-0.09,0.76)$ & 0.102 \\
\hline Hepatic fat (MRI-PDFF) ${ }^{\mathrm{C}}$ & $0.13(-0.28,0.50)$ & 0.532 & $-0.14(-0.71,0.54)$ & 0.718 & $0.01(-0.5,0.52)$ & 0.965 \\
\hline $\operatorname{ALT}(\mathrm{U} / \mathrm{L})$ & $0.39(0.01,0.67)$ & 0.049 & $0.13(-0.54,0.07)$ & 0.731 & $0.51(0.02,0.80)$ & 0.043 \\
\hline AST (U/L) & $0.23(-0.17,0.57)$ & 0.262 & $0.02(-0.62,0.64)$ & 0.953 & $0.19(-0.34,0.63)$ & 0.491 \\
\hline GGT (mg/dL) & $0.26(-0.14,0.59)$ & 0.204 & $0.21(-0.48,0.74)$ & 0.575 & $-0.06(-0.54,0.45)$ & 0.836 \\
\hline Triglycerides (mg/dL) & $0.24(-0.16,0.58)$ & 0.235 & $0.25(-0.45,0.76)$ & 0.499 & $-0.03(-0.51,0.48)$ & 0.928 \\
\hline Total-C (mg/dL) & $0.19(-0.21,0.54)$ & 0.352 & $-0.05(-0.66,0.6)$ & 0.888 & $0.16(-0.37,0.61)$ & 0.567 \\
\hline LDL-C (mg/dL) & $0.16(-0.24,0.51)$ & 0.442 & $-0.18(-0.73,0.5)$ & 0.623 & $0.32(-0.21,0.7)$ & 0.239 \\
\hline HDL-C (mg/dL) & $0.28(-0.12,0.60)$ & 0.168 & $0.45(-0.25,0.84)$ & 0.195 & $0.10(-0.42,0.57)$ & 0.715 \\
\hline Weight (kg) & $0.45(0.08,0.72)$ & 0.019 & $0.38(-0.33,0.81)$ & 0.290 & $0.40(-0.12,0.75)$ & 0.130 \\
\hline
\end{tabular}

${ }^{A}$ In the control group, 2 participants were missing de novo lipogenesis (DNL) data at week 8 and 1 had an outlier value for percentage change in DNL $(+480 \%)$ and were excluded from analyses. ${ }^{B}$ Estimated based on Pearson's correlation. Bold values indicate significant correlations $(P<0.05)$. ${ }^{\complement}$ In the treatment group, 1 participant was missing magnetic resonance imaging-proton density fat fraction (MRI-PDFF) data at week 8 and was excluded from analyses in this row. In the treatment group, 1 participant was missing insulin data at week 0 and was excluded from analyses in this row. TEI, total energy intake; ALT, alanine aminotransferase; AST, aspartate aminotransferase; GGT, $\gamma$-glutamyl transferase; Total-C, total cholesterol; LDL-C, low-density lipoprotein cholesterol; HDL-C, high-density lipoprotein cholesterol.

results support the notion that these metabolic exposures may also be driving increased DNL in individuals with NAFLD.

It should be noted that recent studies in mice have uncovered a potential role of the small intestine in initial phosphorylation of oral fructose, particularly at low doses $(35,36)$. The typical oral intake of fructose in sweetened beverages, however, greatly exceeds the low doses that were tested in mice (which were equivalent to 1-2 ounces of a typical soft drink in a human subject; ref. 35), so this revised metabolic model would not alter the metabolic explanation for reduced free-sugar intake that we observed.

We also found that while percentage changes in hepatic fat (measured by MRI-PDFF) and hepatic DNL were both consistently decreased by the intervention, there was no correlation between their magnitude of changes. This suggests that the relationship between hepatic fat and DNL may not follow a linear, dose-response relationship and/or that there may have been intermediary factors that explain individual differences in the relationship between DNL and hepatic fat. For example, although weight loss was not intended, some participants in the treatment group lost a small amount of weight and weight change was directly correlated with change in DNL, as well as hepatic fat $(r=0.58, P=0.002)$. Additional studies in which weight is more tightly controlled are needed to further understand the relationship between DNL and hepatic fat in youth, independent of weight loss. Notably, change in DNL was associated with change in ALT, which is associated with histological severity of NAFLD (37) and is used as a noninvasive biomarker of hepatic inflammation (38). This correlation suggests that there may also be a direct link between hepatic DNL and other components of NAFLD severity, beyond hepatic fat accumulation.

This study has both limitations and strengths. To perform a repeat measurement of DNL within 8 weeks, it was necessary to correct for any residual isotopic enrichment that is still present in hepatic TG from the baseline labeling period and that carried over to the second labeling period. The central idea in performing this correction is to subtract any residual label in sampled TG still present from the first labeling period. This first involves measuring residual label in blood TG immediately before the repeat heavy water administration. However, some of the residual label present at the beginning of the second labeling period will die away during the 7-day period of repeat labeling. Accordingly, we also had to calculate how much residual label would have remained after this 7-day die-away period to be properly subtracted from the final value. We have elsewhere developed an algorithm for correcting the contribution of baseline labeling in repeated DNL measurement studies in adults with NASH (refs. 12, 14; K.W. Li and M.K. Hellerstein, unpublished observations; Jay Chuang [Gilead], Andrew Billin [Gilead], Rob Myers [Gilead], and Chuhan Chung [Gilead], personal communication). This algorithm requires measuring the amount of residual isotopic label in plasma palmitate at the beginning of the second labeling period and then estimating its turnover rate during the subsequent period of labeling (the turnover rate is calculated from additional blood samples). For the current study, we made 2 simplifications to this algorithm to reduce the number of blood draws in our pediatric participants. First, we elected to use a dried blood spot on day 47 to sample the residual label in plasma TG palmitate at the start of the second labeling period. Reassuringly, measurements of DNL from blood spots correlated closely with those from plasma samples (Supplemental Figure 2), which allowed the dried blood spot results to be used as the initial residual label value. Second, sensitivity analysis showed that varying the turnover rate of hepatic TG palmitate over the 7-day labeling period only altered calculated DNL values by less than $2 \%$. Thus, 
A

Full sample $(n=26)$
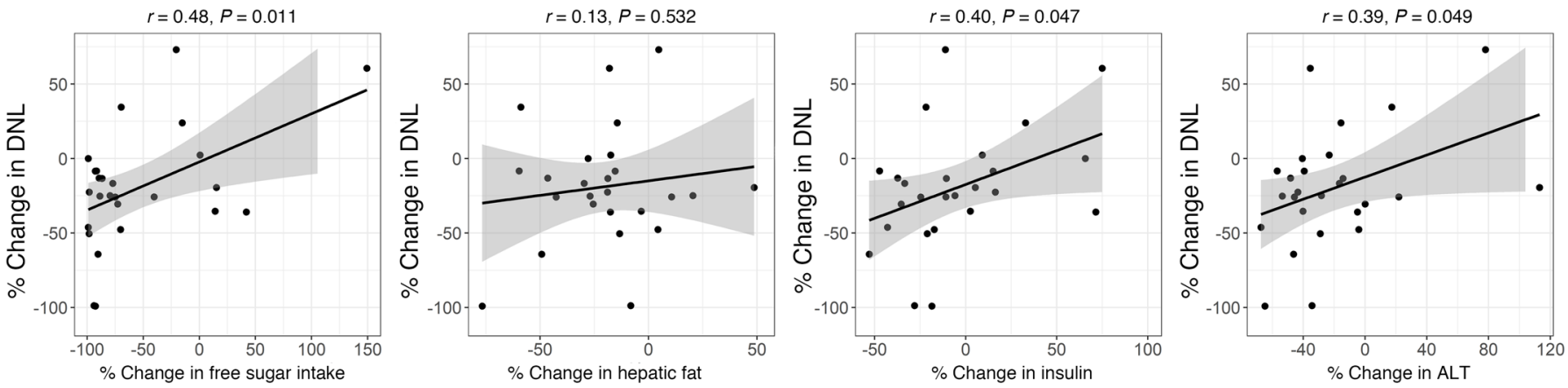

B

Treatment group $(n=16)$
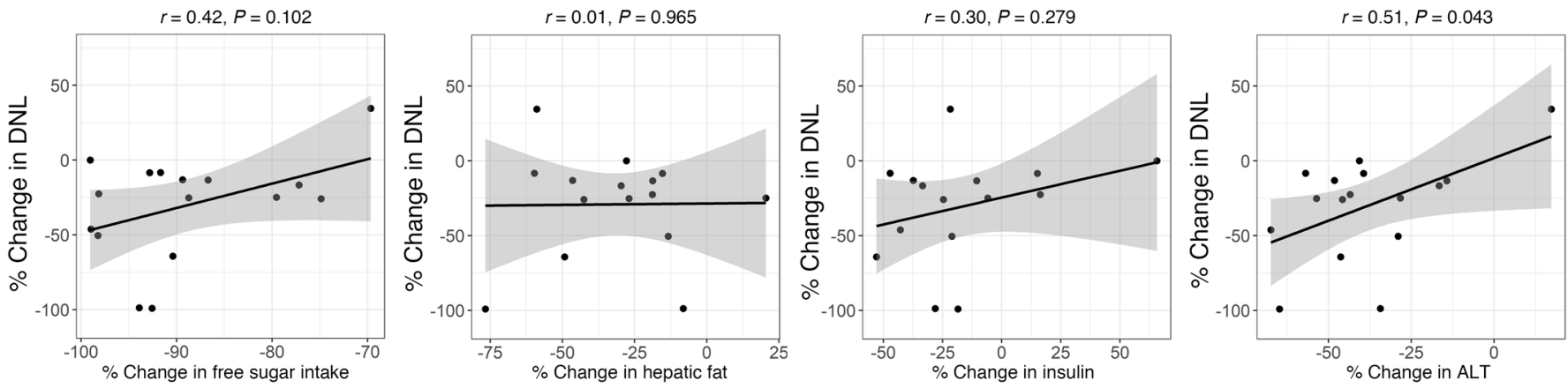

Figure 4. Scatter plots showing correlations between percentage change in hepatic DNL and free-sugar intake, hepatic fat (MRI-PDFF), fasting insulin, and ALT after the 8-week interventions. (A) Full sample. (B) Treatment group. Correlation coefficients and $P$ values were calculated based on Pearson's correlations. Trend lines and 95\% confidence intervals were calculated by linear regression (method = "Im" in the ggplot2 package of R). In the control group, 2 participants were missing DNL data at week 8 and 1 participant had an outlier value for percentage change DNL (+480\%) and were excluded from all plots. In the treatment group, 1 participant was missing hepatic fat (MRI-PDFF) data at week 8 and 1 participant was missing insulin data at week 0 and both were excluded from specific plots. MRI-PDFF, magnetic resonance imaging-proton density fat fraction; ALT, alanine aminotransferase.

it was not necessary to measure this parameter in individual participants under the 7-day labeling study design used. Instead, we applied the average hepatic TG palmitate half-life previously determined in large studies with heavy water metabolic labeling in adults (refs. 12, 24; Lawitz, unpublished observations). The fact that we did not observe a systematic time-related difference in calculated DNL during the repeat labeling period compared to baseline DNL among participants on the control diet, in whom we also used this same approach to correct for the residual label, supports the validity of this approach. We should also note that the day 47 dried blood spot was nonfasted, which may lead to an underestimation of the residual label value if there were dilution due to other sources of TGs in the fed state. This would mean our calculation of percentage hepatic DNL at week 8, after accounting for the residual label, may be higher than if we used a fasted dried blood spot. Accordingly, the net effect would trend against the conclusions of this study by providing a more conservative estimate of the effect of the intervention on hepatic DNL in the treatment group, but similar overall conclusions.

Other limitations were that the sample only included boys with NAFLD, which limits the generalizability of the findings to girls, who may exhibit sex-specific differences in DNL compared with boys (39). In addition, 11 participants from the parent study declined to participate in the substudy, which decreased our power. Future studies with a larger and diverse sample are needed to confirm these findings and conduct adequately powered stratified analyses. No adjustments were made for pubertal stage during the intervention, which may influence NAFLD severity; however, given it is unlikely that participants changed pubertal stage over the 8-week treatment and since comparisons were based on repeated (within participant) measures, we believe this had little impact on our findings. We relied on self-reported data for assessing dietary free-sugar intakes at baseline and week 8 , which are subject to recall and social desirability biases (40-42); however, for the treatment group, these were reported in the setting of a tightly controlled food provision trial, increasing the likelihood of accuracy. Lastly, as noted earlier, the rates of hepatic DNL in our sample, especially at baseline, are consistent with published values in large numbers of adults with fatty liver disease or NASH (12, 24). Additional studies aiming to replicate our findings in children with elevated hepatic fat, who were not also preselected for high sugar intake, will be of interest.

The study was strengthened by the use of 7-day in vivo stable isotope labeling to assess hepatic DNL. This provides a quantitative, time-integrated, and cumulative measurement of hepatic lipogenesis throughout the circadian cycle over 1 week 
in free-living subjects. We also used MRI-PDFF, a precise biomarker of fat content that has been validated against magnetic resonance spectroscopy (43), to accurately quantify hepatic fat at baseline and study completion. We performed multiple 24-hour dietary recalls using a multiple-pass method at each time point to assess dietary intake, which reduces potential measurement error. Finally, the intervention in this study involved the provision of all foods and beverages to the participants and their families in the dietary treatment group. This helped to ensure that dietary free sugar was reduced to the a priori goal ( $<3 \%$ of energy intake) and that participants were compliant with a low-sugar diet throughout the 8-week duration.

In this study, 8 weeks of consuming a diet low in free sugar, compared with the participants' usual diet, was associated with a significant reduction in hepatic DNL among adolescent boys with NAFLD who were regular consumers of sugar-sweetened beverages at baseline. The correlations between hepatic DNL, freesugar intake, and fasting insulin suggest that the reduction in DNL was driven by decreased hepatic sugar and/or insulin exposure. The parallel changes in DNL and hepatic fat (MRI-PDFF), as well as ALT, which all decreased over the 8-week intervention among treatment group participants, is consistent with the hypothesis that hepatic DNL is a pathogenic factor linking high dietary sugar intake with pediatric NAFLD risk, whereas the lack of a strong correlation between changes in DNL and hepatic fat in individual participants suggests that other metabolic processes in addition to DNL, such as the delivery or oxidation of fatty acids in liver, may also be important in modulating hepatic fat content during the dietary intervention.

\section{Methods}

Study population. The study population included adolescent boys, 11 to 16 years old, with biopsy-proven NAFLD who were randomized into a low-free-sugar diet treatment group or control group (their usual diet) for 8 weeks (23). The original randomized controlled trial was completed at 2 academic clinical research centers in the United States (Emory University and UCSD) from August 2015 to July 2017. Details of the dietary treatment are described below. The decision to include only boys was based on population studies showing that pediatric NAFLD prevalence is higher in boys compared with girls (44). In addition, puberty has been shown to modify NAFLD severity (45) and girls tend to undergo pubertal onset earlier than boys, which could confound the effects of the treatment. In lieu of increasing the sample size, we therefore decided to study boys only for this pilot intervention study. Eligibility criteria for the study included a clinical-pathological diagnosis of NAFLD by liver histology, presence of hepatic steatosis measured by MRI-PDFF of $10 \%$ or greater, ALT of $45 \mathrm{U} / \mathrm{L}$ or greater, and current sugar-sweetened beverage or juice consumption of at least 3 servings/week of 8 fluid ounces (23). A total of 40 participants completed the parent clinical trial and, among these participants, 11 declined to participate in the DNL substudy. Therefore, the sample for this study consisted of 29 participants (16 in the treatment group and 13 in the control group) who completed the DNL substudy using stable isotope tracers at baseline and/or study completion (Figure 1). The handling of missing data for hepatic DNL is described in the Statistics section below.

Dietary treatment. Details of the intervention diet were previously described by Schwimmer et al. (23). Briefly, participants were random- ized to either the treatment group or control group after screening at a 1:1 ratio. Participants and investigators were not blinded (open label) due to the impracticality of blinding diets. In the treatment group, home visits were conducted prior to initiation of the intervention to assess habitual diet, dietary preferences, and weekly food volume for the participant and their family. All products containing free sugar (defined as sugars added to foods and beverages and the sugar in fruit juice) were removed from the home and replaced with no-sugar or low-free-sugar substitutes. On a weekly basis, meal plans were created in collaboration with a registered dietitian to match the habitual diet of the participant, but within the goal of less than $3 \%$ of calories from free sugar. All foods and beverages were purchased by the research staff or prepared by the metabolic kitchen, and were provided to the participant and their family for 8 weeks. Adherence to the dietary treatment was ensured through twice-weekly phone calls with research staff. In the control group, participants remained on their usual diet and were provided a weekly food stipend to be used at the retailer of their choice for 8 weeks.

Dietary assessment. Three 24-hour dietary recalls (2 weekdays and 1 weekend day) were collected from each participant before baseline and at study completion by registered dietitians or trained research coordinators using the Nutrition Data System for Research (NDSR) (2015 version, University of Minnesota) and the multiple-pass approach. The data were analyzed in NDSR to calculate total energy intake (in kilocalories/day) and total intake of free sugars (in grams/ day). Intake of free sugars was then converted to a percentage of total energy intake (TEI) by dividing by total kilocalories per day and then averaged across the 3 days, both at baseline and at study completion.

Anthropometric and clinical assessments. Height and weight were measured at baseline and study completion, and used to calculate age- and sex-adjusted body mass index (BMI) percentiles and $z$ scores using the CDC 2000 Growth Charts (46). Waist circumference (based on the top of the iliac crest) and hip circumference were measured at baseline and study completion for all participants. Hepatic fat was measured at baseline and study completion by MRIPDFF following a fast of at least 4 hours. This was performed using an advanced magnitude-based, spoiled-gradient-echo MRI-PDFF estimation technique, which has been previously validated to measure hepatic steatosis in children (47), as described in detail in the original intervention study (23). Fasting blood specimens were also collected at baseline and week 8 , and used to measure laboratory values, including fasting glucose and insulin, liver enzymes, and blood lipids, by standard clinical assays.

DNL assessment by use of metabolic labeling with stable isotope tracers. Participants initiated the stable isotope metabolic labeling protocol during a study visit approximately 1 week prior to randomization (nominally day -7) and were instructed to consume $50 \mathrm{~mL}$ of heavy water $\left(70 \%{ }^{2} \mathrm{H}_{2} \mathrm{O}\right)$ twice a day for up to 7 days. A similar labeling protocol has been shown in adults to produce a steady ramp in body water enrichments to a peak on day 7 of $1.0 \%-2.0 \%{ }^{2} \mathrm{H}_{2} \mathrm{O}(24)$. On day 0 (baseline visit), tracer administration was stopped and a fasting blood sample and a dried finger-stick blood spot sample on filter paper were collected. Across the 29 participants, the mean \pm SD for deuterium oxide enrichment after 7 days of baseline labeling was $0.79 \% \pm 0.4 \%$ molar percent excess. On day 47 , a second dried blood spot was collected during a home visit (nonfasting), and the stable isotope labeling protocol was restarted, in which participants 
again consumed $50 \mathrm{~mL}$ of deuterated water twice a day for 7 days. On day 56 (final study visit), another fasting blood sample and dried blood spot were collected. A summary of the study design, including the timing of the stable isotope labeling protocol for DNL measurements, is shown in Figure 2.

Determination of hepatic DNL in plasma TG palmitate. The fractional contribution of hepatic DNL to TG palmitate in plasma was determined at the University of California, Berkeley in blood samples and dried blood spots collected during periods of heavy water exposure. Palmitate from plasma TG was esterified and analyzed for mass isotopomer abundances by gas chromatography-mass spectroscopy (GC/MS), using mass isotopomer distribution analysis (MIDA) to determine the effective body water deuterium exposure (precursor pool enrichment) for the calculation of fractional DNL, as previously described $(24,48,49)$. Briefly, total lipids were extracted from plasma or blood spot samples with chloroform/methanol (2:1) and the plasma TGs were isolated via thin layer chromatography. Plasma TG fatty acids were transesterified to fatty acid methyl esters for GC/MS analysis to quantify excess isotopic abundance, or enrichment, of the M1 isotopomer (EM1) and the M2isotopomer (EM2) of palmitate due to incorporation of deuterium from body water into stable $\mathrm{C}-\mathrm{H}$ bonds of palmitate, which occurs during synthesis through the DNL pathway (25). The proportion of plasma TG palmitate that originated from the DNL pathway was then calculated from the EM1 and EM2 of palmitate using MIDA to determine both the body water precursor enrichment and the corresponding isotopic enrichment of newly synthesized palmitate molecules. The decision to obtain palmitate measurements from whole plasma, and not after isolation from large verylow-density lipoproteins (VLDLs), was based on prior data from our group showing that isotopic enrichments from total plasma TG palmitate after transesterification is strongly correlated with VLDL-TG enrichments (Supplemental Figure 3).

\section{The calculation of DNL at baseline is given in Equation 1:}

$\% D N L_{B L}=\% E M 1_{t} / \% E M 1^{*}$, (Equation 1 ) where $\% E M 1_{t}$ is the isotopic enrichment in plasma TG palmitate from the plasma sample taken 5-7 days after starting the baseline heavy water labeling. \%EM1* is the maximal or asymptotic (equilibrium) isotopic enrichment in the M1-mass isotopomer in newly synthesized palmitate that could be achieved from the measured integrated heavy water exposure (precursor enrichment), as determined using MIDA (48).

Correction model for residual label at week 8 of dietary intervention. During test/retest metabolic labeling studies, interpretation of the follow-up labeled palmitate enrichment requires correction for potential residual label that is still present from the baseline metabolic labeling protocol. In this study, analysis of the isotopic enrichment in dried blood spot samples collected on day 47, prior to the start of the week 8 labeling period, indicated that extracted TG palmitate contained a significant amount of residual isotopic label (EM1 of $0.48 \% \pm 0.37 \%$; Supplemental Figure $2 \mathrm{~A}$ ), which corresponds to an isotopic enrichment in plasma TG of $0.55 \% \mathrm{EM} 1$, after correction for dilution in dried blood spots (Supplemental Figure 2B). This residual label represents a non-negligible background for any isotopic label incorporated during the subsequent labeling period by DNL, so it is necessary to subtract the contribution from residual label to calculate new DNL. This correction requires an estimate of the turnover rate at which any preexisting labeled palmitate dies away during the repeat labeling period. Specifically, the enrichment from previously labeled palmitate present in liver at the end of the repeat labeling study (nominally 7 days later) needs to be subtracted to accurately determine the label that was newly incorporated during the repeat study. Accordingly, we have developed a method for calculating the rate at which residual labeled TG palmitate in the liver decays during the repeat labeling period. This method requires serial blood draws after cessation of labeling to characterize the die-away kinetics of preexisting labeled TG palmitate. In the current study, to avoid multiple blood draws in children, we did not measure the dieaway of residual label directly in participants, but instead used the average die-away rate of labeled palmitate in plasma that we determined previously in large studies of adult patients with NAFLD and NASH (refs. 12, 14; K.W. Li and M.K. Hellerstein, unpublished observations; Jay Chuang [Gilead], Andrew Billin [Gilead], Rob Myers [Gilead], and Chuhan Chung [Gilead], personal communication), which revealed a half-life of $16.7 \pm 6.3$ days. We also carried out here a sensitivity analysis for error potentially introduced by use of this previously determined value (see below).

\section{This correction for residual label was applied at the week 8 labeling period and was implemented mathematically as in Equation 1:}

$\% D N L_{W 8}=\left(\% E M 1_{t}-\left[\% E M 1_{t 0} \times \exp \left(-t / t_{1 / 2}\right)\right]\right) / \% E M 1^{*},($ Equation 2) where $\% D N L_{w 8}$ is the fractional contribution of new hepatic DNL during week 8, as determined from the increase in isotopic enrichment (EM1) in plasma TG palmitate after repeat labeling with heavy water. $\% E M 1_{t}$ is the isotopic enrichment in plasma TG palmitate from samples taken after the repeat labeling period occurring at week 8 of treatment, which represents the summed combination of the isotopic label from newly synthesized palmitate and the residual isotopic label from the previous labeling period. $\% E M 1_{t 0}$ is the isotopic enrichment in TG palmitate at the beginning of the repeat labeling period (started on study day 47), taken to represent input from the slow turnover storage pool of hepatic TG. \%EM1* is the maximal or asymptotic isotopic enrichment in the M1-mass isotopomer in newly synthesized palmitate that could be achieved from the measured integrated heavy water exposure (precursor pool enrichment), as determined using MIDA (48). $t$ is the time from the start of the repeat labeling period to the repeat blood sample. $t_{1 / 2}$ is the half-life of palmitate in a slow-turnover pool (16.7 days).

After implementing the corrections for the residual label, 1 participant had a negative DNL value at week 8; accordingly, we assumed this value was below the detection limit and was imputed with half the minimum for all measured values. To estimate the error associated with the application of a residual background calculation based on results from adult NAFLD and NASH patients (refs. 12, 14; K.W. Li and M.K. Hellerstein, unpublished observations; Jay Chuang [Gilead], Andrew Billin [Gilead], Rob Myers [Gilead], and Chuhan Chung [Gilead], personal communication), we performed a sensitivity analysis that compared DNL calculated by Equation 2 corrected using the average half-life in adult NAFLD patients of 16.7 days, to DNL calculated using the extremes of the observed range in these subjects ( \pm SD of 6.3 days). The resulting DNL was changed by only $2 \%$ to $4 \%$ in either direction, despite a $38 \%$ variation in half-life (Supplemental Figure 4), indicating that the value of residual label half-life was not a critical part of the correction in this study, where the period during which residual label could further decay was for only 7 days. 
Statistics. Descriptive statistics were performed to examine characteristics of the subsample in this study according to treatment group. Continuous variables were summarized as means and SD or medians and interquartile ranges (IQRs) if normally or non-normally distributed, respectively, and compared between groups using a 2-tailed Student's $t$ test or Mann-Whitney $U$ test, respectively. Categorical variables were summarized as counts and frequencies and compared between groups using $\chi^{2}$ tests. Mixed models were constructed to assess the effect of the diet treatment on percentage contribution from DNL from baseline to week 8. This modeling strategy was chosen because it accounts for the repeated measures of hepatic DNL and is tolerant to missing data, which allowed us to include the 2 participants in the control group who were missing hepatic DNL data at week 8. In the model, treatment group, time, and a group $\times$ time interaction term were fixed effects; participant ID was a random effect and study site was also controlled for as a covariate. Degrees of freedom were estimated using the Kenward-Roger method and standard errors were estimated by an unstructured covariance matrix, which achieved the best model fit based on lowest Aikaike Information Criterion. Results are reported as least-squares (LS) means and 95\% CIs for DNL at baseline and week 8 by treatment group. We also estimated the week 8 mean difference in hepatic DNL between groups, adjusted for baseline, using a conditional joint response model. To evaluate potential confounding by weight loss during the intervention, a post hoc sensitivity analysis was performed in which we additionally adjusted models for percentage weight change as a covariate. Similar mixed models were also constructed to examine the effect of the diet treatment on secondary metabolic outcomes of interest in the subsample who completed the hepatic DNL assessment.

We next calculated the percentage changes for hepatic DNL, hepatic fat (MRI-PDFF), and each secondary metabolic outcome of interest as (week 8 - week 0)/week 0. For this analysis, only participants with complete data at baseline and week 8 were included for relevant analyses. We evaluated associations between percentage change in DNL and percentage change in other variables during the intervention using Pearson's correlations and scatter plots. Given there may be potential effect modification by the dietary treatment, we conducted these analyses both for the full sample and stratified by treatment group. Statistical significance was set at an $\alpha$ of 0.05. All analyses were performed in SAS (v9.4) and all figures were created using the ggplot package in $\mathrm{R}(50)$.
Study approval. Approvals were obtained by the institutional review boards of UCSD and Emory University. Written informed consent from the parent or guardian and assent from all participants were obtained.

\section{Author contributions}

CCC, MBV, JBS, KWL, CB, and MKH designed the study. MBV, JBS, PAUN, JAW, KPN, and CJK designed the original intervention trial. KWL, CB, MD, EMN, and MKH designed the tracer protocol and performed sample analyses. ALA, CAC, RLC, JKS, and CBS assisted with data acquisition and provided administrative or technical support. CCC performed data analyses. JF provided statistical support. CCC, KWL, CB, MKH, and MBV interpreted the data and wrote the manuscript. MBV and JBS acquired funding. All authors critically reviewed and edited the manuscript, and approve of the final manuscript.

\section{Acknowledgments}

The authors thank the study subjects and their families for their participation. The authors also acknowledge Scott Turner for his assistance in designing the stable isotope tracer study and in the correlation study of DNL in VLDL-TG and plasma TG. The authors also acknowledge the research coordinators, staff, and technicians who were involved in carrying out the intervention and data collection. This study was supported by foundational grant funding from the Nutrition Science Initiative (made possible by gifts from the Laura and John Arnold Foundation, Ambrose Monell Foundation, and individual donors); support from the UCSD Altman Clinical and Translational Research Institute; grants UL1TR001442 and UL1TR002378 from the NIH; support from the Children's Healthcare of Atlanta and Emory University's Children's Clinical and Translational Discovery Core; support from the Children's Healthcare of Atlanta and Emory University Pediatric Biostatistical Core; and support from the Georgia Clinical and Translational Science Alliance. CCC is currently supported by NIH National Institute of Diabetes, Digestive, and Kidney Diseases grant no. T32DK07658.

Address correspondence to: Miriam B. Vos, MD, MSPH, Department of Pediatrics, Emory University, 1760 Haygood Drive NE, Atlanta, Georgia 30322, USA. Phone: 404.727.9930; Email: mvos@emory.edu.
1. Welsh JA, et al. Increasing prevalence of nonalcoholic fatty liver disease among United States adolescents, 1988-1994 to 2007-2010. J Pediatr. 2013;162(3):496-500.

2. Sahota AK, et al. Incidence of nonalcoholic fatty liver disease in children: 2009-2018. Pediatrics. 2020;146(6):e20200771.

3. Cali AM, Caprio S. Ectopic fat deposition and the metabolic syndrome in obese children and adolescents. Horm Res. 2009;71 suppl 1:2-7.

4. D'Adamo E, et al. Central role of fatty liver in the pathogenesis of insulin resistance in obese adolescents. Diabetes Care. 2010;33(8):1817-1822.

5. Toledo-Corral CM, et al. Ectopic fat deposition in prediabetic overweight and obese minority adolescents. J Clin Endocrinol Metab.
2013;98(3):1115-1121.

6. Harlow KE, et al. Clinically actionable hypercholesterolemia and hypertriglyceridemia in children with nonalcoholic fatty liver disease. JPediatr. 2018;198:76-83.

7. Pacifico L, et al. Nonalcoholic fatty liver disease and carotid atherosclerosis in children. Pediatr Res. 2008;63(4):423-427.

8. Newton KP, et al. Prevalence of prediabetes and type 2 diabetes in children with nonalcoholic fatty liver disease. JAMA Pediatr. 2016;170(10):e161971.

9. Cioffi CE, et al. Natural history of NAFLD diagnosed in childhood: a single-center study. Children (Basel). 2017;4(5):34.

10. Feldstein AE, et al. The natural history of non- alcoholic fatty liver disease in children: a follow-up study for up to 20 years. Gut. 2009;58(11):1538-1544.

11. Lambert JE, et al. Increased de novo lipogenesis is a distinct characteristic of individuals with nonalcoholic fatty liver disease. Gastroenterology. 2014;146(3):726-735.

12. Smith GI, et al. Insulin resistance drives hepatic de novo lipogenesis in nonalcoholic fatty liver disease. J Clin Invest. 2020;130(3):1453-1460.

13. Chiu S, et al. Dietary carbohydrates and fatty liver disease: de novo lipogenesis. Curr Opin Clin Nutr Metab Care. 2018;21(4):277-282.

14. Jensen T, et al. Fructose and sugar: a major mediator of non-alcoholic fatty liver disease. J Hepatol. 2018;68(5):1063-1075. 
15. Softic S, et al. Role of dietary fructose and hepatic de novo lipogenesis in fatty liver disease. Dig Dis Sci. 2016;61(5):1282-1293.

16. Schwarz JM, et al. Short-term alterations in carbohydrate energy intake in humans. Striking effects on hepatic glucose production, de novo lipogenesis, lipolysis, and whole-body fuel selection. J Clin Invest. 1995;96(6):2735-2743.

17. Parks EJ, et al. Dietary sugars stimulate fatty acid synthesis in adults. J Nutr. 2008;138(6):1039-1046.

18. Stanhope KL, et al. Consuming fructose-sweetened, not glucose-sweetened, beverages increases visceral adiposity and lipids and decreases insulin sensitivity in overweight/obese humans. JClin Invest. 2009;119(5):1322-1334.

19. Beysen C, et al. Dose-dependent quantitative effects of acute fructose administration on hepatic de novo lipogenesis in healthy humans. Am J Physiol Endocrinol Metab. 2018;315(1):E126-E132.

20. Geidl-Flueck B, et al. Fructose- and sucrose- but not glucose-sweetened beverages promote hepatic de novo lipogenesis: a randomized controlled trial. J Hepatol. 2021;75(1):46-54.

21. Schwarz JM, et al. Effect of a high-fructose weight-maintaining diet on lipogenesis and liver fat. J Clin Endocrinol Metab. 2015;100(6):2434-2442.

22. Schwarz JM, et al. Effects of dietary fructose restriction on liver fat, de novo lipogenesis, and insulin kinetics in children with obesity. Gastroenterology. 2017;153(3):743-752.

23. Schwimmer JB, et al. Effect of a low free sugar diet vs usual diet on nonalcoholic fatty liver disease in adolescent boys: a randomized clinical trial. JAMA. 2019;321(3):256-265.

24. Lawitz EJ, et al. Acetyl-CoA carboxylase inhibitor GS-0976 for 12 weeks reduces hepatic de novo lipogenesis and steatosis in patients with nonalcoholic steatohepatitis. Clin Gastroenterol Hepatol. 2018;16(12):1983-1991.

25. Hellerstein MK, et al. Measurement of de novo hepatic lipogenesis in humans using stable isotopes. J Clin Invest. 1991;87(5):1841-1852.

26. McGarry JD, Foster DW. Regulation of hepatic fatty acid oxidation and ketone body production. Annu Rev Biochem. 1980;49:395-420.

27. Santoro N, et al. Hepatic de novo lipogenesis in obese youth is modulated by a common variant in the GCKR gene. JClin Endocrinol Metab. 2015;100(8):E1125-E1132.

28. Schwarz JM, et al. Effects of dietary fructose restriction on liver fat, de novo lipogenesis, and insulin kinetics in children with obesity. Gastroenterology. 2017;153(3):743-752.

29. Hudgins LC, et al. A dual sugar challenge test for lipogenic sensitivity to dietary fructose. J Clin Endocrinol Metab. 2011;96(3):861-868.

30. Ferrannini E, et al. The disposal of an oral glucose load in healthy subjects. A quantitative study. Diabetes. 1985;34(6):580-588.

31. Mari A, et al. Glucose absorption and production following oral glucose: comparison of compartmental and arteriovenous-difference methods. Metabolism. 1994;43(11):1419-1425.

32. Mayes PA. Intermediary metabolism of fructose. Am J Clin Nutr. 1993;58(5 suppl):754S-765S.

33. Kim M-S, et al. ChREBP regulates fructoseinduced glucose production independently of insulin signaling. J Clin Invest. 2016;126(11):4372-4386.

34. Ter Horst KW, Serlie MJ. Fructose consumption, lipogenesis, and non-alcoholic fatty liver disease. Nutrients. 2017;9(9):981.

35. Hellerstein M. Surprising findings in a 'wellunderstood' nutrient-assimilation pathway. Nat Metab. 2020;2(7):561-563.

36. Jang C, et al. The small intestine shields the liver from fructose-induced steatosis. Nat Metab. 2020;2(7):586-593.

37. Patton HM, et al. Clinical correlates of histopathology in pediatric nonalcoholic steatohepatitis. Gastroenterology. 2008;135(6):1961-1971.

38. Lavine JE, et al. Effect of vitamin E or metformin for treatment of nonalcoholic fatty liver disease in children and adolescents: the TONIC randomized controlled trial. JAMA. 2011;305(16):1959-1968.

39. Pramfalk C, et al. Sex-specific differences in hepatic fat oxidation and synthesis may explain the higher propensity for NAFLD in men. JClin Endocrinol Metab. 2015;100(12):4425-4433.

40. Hebert JR, et al. Social desirability bias in dietary self-report may compromise the validity of dietary intake measures. Int J Epidemiol. 1995;24(2):389-398.

41. Heitmann BL, Lissner L. Dietary underreporting by obese individuals--is it specific or non-specific? BMJ. 1995;311(7011):986-989.

42. Schoeller DA. Limitations in the assessment of dietary energy intake by self-report. Metabolism. 1995;44(2 suppl 2):18-22.

43. Yokoo T, et al. Estimation of hepatic proton-density fat fraction by using MR imaging at $3.0 \mathrm{~T}$. Radiology. 2011;258(3):749-759.

44. Anderson EL, et al. The prevalence of non-alcoholic fatty liver disease in children and adolescents: a systematic review and meta-analysis. PLoS One. 2015;10(10):e0140908.

45. Suzuki A, et al. Association between puberty and features of nonalcoholic fatty liver disease. Clin Gastroenterol Hepatol. 2012;10(7):786-794.

46. Kuczmarski RJ, et al. CDC growth charts: United States. Adv Data. 2000;(314):1-27.

47. Schwimmer JB, et al. Magnetic resonance imaging and liver histology as biomarkers of hepatic steatosis in children with nonalcoholic fatty liver disease. Hepatology . 2015;61(6):1887-1895.

48. Hellerstein MK, Neese RA. Mass isotopomer distribution analysis at eight years: theoretical, analytic, and experimental considerations. Am J Physiol.1999;276(6 pt 1):E1146-E1170.

49. Gardner JL, et al. Measurement of liver collagen synthesis by heavy water labeling: effects of profibrotic toxicants and antifibrotic interventions. Am J Physiol Gastrointest Liver Physiol. 2007;292(6):G1695-G1705.

50. The R Project for Statistical Computing. Version 4.1.2. The R Foundation; 2021. Accessed November 2, 2021. https://www.r-project.org/. 